\title{
SISTEMATIZACIÓN DEL PROCESO DE PROSPECTIVA TERRI- TORIAL CONSTRUIDO EN EL HUILA, DURANTE EL PERIODO 1990-2010 CON EL SOPORTE DE LA UNIVERSIDAD SURCOLOMBIANA
}

\section{SYSTEMATIZATION OF TERRITORIAL FORESIGHT PROCESS BUILT IN HUILA DURING THE PERIOD 1990-2010 WITH THE SUPPORT OF UNIVERSIDAD SURCOLOMBIANA}

«Es absurdo continuar haciendo lo mismo y esperar resultados diferentes».

Pensamiento Chino

Sergio Alexander Santos Sánchez*, Carlos Eduardo Amézquita Parra**, Humberto Rueda Ramírez ${ }^{\star \star \star}$

\section{Resumen}

La Prospectiva Estratégica es considerada como una disciplina científica que estudia el futuro con el fin de diagnosticarlo y construirlo desde el presente. Algunos autores la denominan la ciencia de la esperanza. Según este enfoque, el «visionar estratégicamente un territorio» (construyendo escenarios futuros) se le conoce como Prospectiva Territorial.

Varios son los ejercicios de futuro que se han realizado en el Territorio del Huila; tales como el Plan Integral de Neiva (PIDNE II: 1990-2015), la VISION Huila 2020, el Plan Estratégico de Ciencia, Tecnología e Innovación del Huila: 2005-2020, el Plan Decenal de Cultura del Huila 2011-2020, entre otros, los cuales han proporcionado líneas estratégicas para la Planificación de su futuro en el 2020.

En este contexto histórico, por tanto objetivo y metodológico, el sistematizar las más recientes experiencias prospectivas del Huila posibilita identificar las intencionalidades que son comunes en la sociedad huilense como también la ruta estratégica posible contenida en ellos, hacia el año 2020.

Palabras clave: Estudios de futuro, prospectiva estratégica, pensamiento estratégico, prospectiva territorial.

\section{Abstract}

The strategic foresight is regarded as a scientific discipline that studies the future in order to diagnose it and build it from the present. Some authors call it the science of hope.

Artículo recibido: 20/06/2013 Aprobado: 14/07/2013

* (C) Magíster en Pensamiento Estratégico y Prospectiva. Investigador Grupo Simbiosis, Hombre y Naturaleza de la Universidad Surcolombiana. Email: sealsa@hotmail.com

** Magíster en Dirección Universitaria. Investigador del Grupo de Investigación Simbiosis Hombre y Naturaleza. Email: cedamez@usco.edu.co

*** Magíster en Dirección Universitaria. Director del Grupo de Investigación Simbiosis Hombre y Naturaleza. Email: humrueda@gmail.com 
Under this approach, "the process of creating a strategic vision of a territory" (building future scenarios), is called Territorial Foresight. There are several prospective exercises that have been carried out in the territory of Huila; such as the Comprehensive Plan of Neiva (PIDNE II: 1990-2015), the Huila 2020 VISION, the Strategic Plan for Science, Technology and Innovation of Huila: 2005-2020, the ten-year Plan of Culture of Huila 2011-2020, among others, which have provided strategic lines for the planning of Huila's future in 2020 .

Key words: Future Studies, Strategic Foresight, Strategic Thinking, Territorial Prospective

El Departamento de Huila tiene una superficie de 19.890 $\mathrm{km}^{2}$ que representa el $1,75 \%$ del territorio nacional, una población aproximada de 1.000 .000 de habitantes, (según Censo General de Colombia 2005) y está situado al sur de la región Andina de Colombia o región del Alto Magdalena (sur del Río Magdalena), en las siguientes coordenadas geográficas: latitud $4^{0} 35^{\prime} 56.57 »$, longitud $74^{\circ} 4^{\prime} 51.30 »$ al Oeste de Greenwich. Limita por el Norte con Bogotá(Capital de la Nación) y los Departamentos del Tolima y Cundinamarca, por el Este con Meta y Caquetá, por el Sur con Caquetá y Cauca, y por el Oeste con Cauca y Tolima. El Río Magdalena es la vertiente hidrográfica más importante de Colombia.

\section{Introducción}

Pensar y conocer el futuro anticipadamente ha sido parte fundamental en la evolución de las distintas civilizaciones a través de la historia; igualmente han sido diversas las formas de aproximarse a él. Ya en el siglo XX surgen numerosos autores con novedosos enfoques, los cuales logran sentar las bases conceptuales de los llamados estudios de futuro.

En Francia nace la Prospectiva como la ciencia que estudia el futuro para comprenderlo y construirlo, fueron Ios filósofos Mauricio Blondel y Gastón Berger los principales precursores quienes dieron a conocer esta nueva ciencia por toda Europa y el resto del mundo. Años después, surge el francés Michel Godet, quien aporta un modelo a la prospectiva y la define a como la ciencia que estudia el futuro para poder influir en el.

En otras palabras, la Prospectiva Estratégica es la nueva forma de planificar en la sociedad del conocimiento; implica cambios en la forma de pensar, de ver y entender los fenómenos que suceden alrededor nuestro y, lo más importante, tener la visión de largo plazo para lograr transformar el futuro conjeturado con las acciones del presente.
Finalmente, los autores del Artículo advierten que buscando la objetividad necesaria, y fieles a las Metodologías practicadas en los distintos momentos de la Prospectiva Territorial del Huila (las de la Construcción Social Regional, la Investigación Acción Participativa, como las de la Acción Comunicativa) se han respetado al máximo los deseos y las propuestas concertadas con los Actores Sociales en cada episodio.

\section{Evolución de la prospectiva estratégica}

Desde sus inicios a la humanidad siempre le ha atraído e intrigado conocer el futuro; en diferentes épocas de la historia y en diversas civilizaciones han surgido numerosas formas de representarlo; en el contexto mágico y religioso surgieron la adivinación, siendo las más conocidas las pitonisas del Oráculo de Delfos en la antigua Grecia; y las profecías, como las de la cultura Maya en América y posteriormente las de Nostradamus e Isaac Newton en Europa.

Los primeros historiadores hicieron compilaciones de relatos y epopeyas que provenían de la tradición oral y escrita, verdaderos gestores de los actuales estudios de futuro. Platón, por ejemplo, en el siglo V (A. de C.) propone en «La República» un concepto filosófico del Estado ideal 0 utópico.

Años después, Tomas Moro en su obra "Utopía» (1516), complementa lo expuesto por Platón, la organización de sociedad ficticia pero a su vez con ideales filosóficos y políticos, estableciendo el bien de la comunidad como factor determinante en las pretensiones particulares.

En 1798, Thomas Malthus publica el libro «Ensayo sobre el Principio de la Población»; utiliza las matemáticas para hacer predicciones sobre el crecimiento futuro de la población. 
Posteriormente, a finales del siglo XIX, se propone a la ciencia ficción como la nueva forma de representar el futuro: Julio Verne con obras como «Veinte Mil Leguas de Viaje Submarino», «De la Tierra a la Luna»,» Viaje al Centro de la Tierra», entre otros; y Herber George Wells con «La Máquina del Tiempo», «La Guerra de los Mundos», «El Hombre Invisible», son los creadores de la ciencia ficción.

A partir de estos antecedentes remotos, la necesidad de tener estudios de futuro va emergiendo gradualmente a nivel mundial y se va consolidando a lo largo del siglo XX como aquel campo del conocimiento de la planificación del largo plazo. Precisamente, es en esta época que surgen las dos principales escuelas contemporáneas de Prospectiva, una en Europa y otra en América.

Gastón Berger (Francia) denomina a esta disciplina «la prospectiva», palabra del verbo latino «prospicere» que significa «ver hacia adelante» no tiene la finalidad de predecir el futuro ni de adivinarlo, su objeto principal es construirlo.

En Estados Unidos, el estudio de futuro se origina a finales de la década de 1940 con la creación de la «Rand Corporation» quien desarrolla años más tarde métodos prospectivos como la «Técnica Delphi», utilizada especialmente para reconocer el cambio en virtud de la fuerza de las tendencias y por lo tanto se comprende la realidad de una manera lineal. A esta corriente se le conoce como «forecasting» 0 «pronóstico» al traducirse al español.

En la década de 1970 el francés Michel Godet, establece un modelo con fundamentos matemáticos que da origen a la «Prospectiva Estratégica»; tiene como propósito estudiar el futuro para poder actuar en el presente con mayor seguridad.

De otra parte, el Pensamiento Estratégico evoluciona paralelamente y se define «Como la capacidad para resolver con éxito los problemas en forma individual y colectiva, vale decir, para asumir una tarea de alto nivel de complejidad, tomar decisiones estratégicas con autonomía (o sin una guía o supervisión externa muy cercana) y responsabilizarse por sus resultados en el futuro». Mant (Citado por Medina y Ortegón, 2006, p. 91).

Medina y Ortegón (2006), afirman que el Pensamiento Estratégico es el «proceso de razonamiento acerca de sistemas o problemas complejos, con miras a lograr un objetivo» (p. 91).
En esta misma dirección conceptual la Prospectiva y el Pensamiento Estratégico resultan ser complementarios, la forma más conveniente para incidir el entorno organizacional con decisiones y acciones elegidas desde el presente (Godet). Incluso, dentro de las principales aplicaciones del enfoque de la Prospectiva se encuentra el de la Prospectiva Territorial, se define como el proceso sistemático desarrollado por los actores sociales para «poder visionar» un «territorio en el largo plazo» con el fin de desarrollarlo y/o transformarlo estratégicamente, es decir, en el futuro.

La reflexión que desarrollamos en el presente artículo tiene como propósito central demostrar la vigencia y aplicabilidad de la Prospectiva Territorial en un complejo pero específico espacio territorial, de Colombia, en el Departamento del Huila. Comprender la lógica y manejo de un Territorio Complejo por su Biodiversidad (humana y no-humana) y por la intensidad de sus conflictos sociales y políticos, nos han llevado a practicar intuitiva y normativamente sucesivos estudios prospectivos desde 1990.

\section{Experiencias y estudios prospectivos en el Huila}

El departamento del Huila ha sostenido una intensa tradición de estudios de futuro soportados en la Prospectiva, quizá predispuesto por disímiles imaginarios culturales desde la ancestral cultura Agustiniana y por el legendario líder empresarial y político Reynaldo Matiz (quien imaginó al Huila como estrella vial de la conexión: Huila - mar Pacífico - mar Atlántico - Amazonia, a finales del siglo XIX) hasta la incitante premisa expuesta por el escritor y líder huilense José Eustasio Rivera en «Huila Tierra de Promisión» (Tierra de Futuro), a mediados del XX.

Recientemente, y durante un largo periodo: 1990-2011 se cumplieron varios ejercicios de planificación prospectiva en territorio huilense con el soporte científico y acompañamiento estratégico de la Universidad Surcolombiana.

En los 6 ejercicios sistematizados a continuación se observa que todos aplicaron el modelo Prospectivo, particularmente en las siguientes experiencias: «HUILA Visión 2020» formulado en 2000; «Prospectiva Estratégica de la Ciencia, Tecnología e Innovación del Huila 
2020» formulado en 2004; la «Red Departamental de Colegios Agropecuarios del Huila» en el 2006, y en 2010 «Plan Decenal de Cultura del Huila 2011-2020». Los 2 ejercicios restantes, aplicaron metodología prospectiva de manera espontánea: el Plan Integral de Neiva (PIDNE II: 1990-2015) y la Prospectiva Huila 2095.

En las experiencias se puede evidenciar que cumplieron con las siguientes fases *:

Elaboración del Estado del Arte: se tuvieron en cuenta aspectos como: el económico, social, cultural, político, ambiental, tecnológicos, etc. En algunos ejercicios los antecedentes se pueden homologar.

Desarrollo de Talleres: en todos los ejercicios se desarrollaron talleres con los Actores sociales involucrados en cada experiencia, así:

- Identificación Factores de Cambio o Críticos: la primera parte es este punto, donde se identifican los factores de cambio, es decir, es donde se establecerán cuáles son los fenómenos que no tenemos certeza de evolución y de aquellos que tenemos mayor certitud de ocurrencia.

- Identificación de Variables Estratégicas: Es la segunda parte del primer taller. En él se identificarán unas variables del taller anterior, las cuales se denominaran variables estratégicas, no todos las variables encontradas en la fase anterior son importantes, pero las escogidas serán las de mayor impacto.

- Construcción de Escenarios: Primera parte. En este punto converge toda la información que se ha recabado hasta el momento, en particular, las ideas que provienen de las tendencias y mejores prácticas mundiales como antecedentes. El escenario es la descripción de una situación futura junto con la progresión de eventos desde la situación base hasta la situación futura.

- Elaboración de Estrategias: Una vez reconocido el o los escenarios «apuesta» se definieron las estrategias para llegar al futuro seleccionado..

A continuación se ilustra el modelo (ver gráfico 1).

A continuación se analizarán brevemente cada uno de los ejercicios prospectivos desarrollados en Huila desde 1990:
Plan Integral de Desarrollo para Neiva - PIDNE (1990-2015)

Durante la década de 1980 ocurrieron en Colombia sucesivos procesos de urbanización que ocasionaron importantes reglamentos urbanos para facilitar el ordenamiento territorial en los municipios y propiciar mejores condiciones para la vida humana. Entre las principales normas promulgadas se pueden mencionar, el Decreto 1306 de 1980 que obliga a los municipios con más de 20.000 habitantes a formular planes integrales de desarrollo, la Ley 11 de 1986 por la cual se dicta el Estatuto Básico de la Administración Municipal y se ordena la participación de la comunidad en el manejo de los asuntos locales, se reforma el Decreto 232 de 1983 y, la Ley 09 de 1989 por la cual se dictan normas sobre planes de desarrollo municipal, compraventa y expropiación de bienes, entre otras normas.

En este contexto $y$, fundamentalmente dando cumplimiento a la Ley 09 de 1989, conocida como «ley de reforma urbana» el alcalde Jairo Morera Lizcano que gobernó la ciudad en el periodo 01-01-1988 a 01-01-1990, debidamente autorizado por el Concejo Municipal, convocó un equipo técnico de profesionales liderado por la Universidad Surcolombiana.

En el artículo 1 de la Ley 09 de 1989 se establece que:

... las ciudades con una población mayor de cien mil (100.000) habitantes, (...), deberán formular su respectivo plan integral de desarrollo de conformidad con la política nacional y departamental, las técnicas modernas de planeación urbana y con base en la coordinación del desarrollo urbano-regional (p. 1).

Conforme a esta directriz el equipo técnico decidió utilizar la prospectiva como herramienta de planificación urbana regional a largo plazo de la ciudad de Neiva (25 años).

Por lo tanto, el «Plan Integral de Desarrollo para Neiva 1990-2015», conocido como PIDNE II, fue el primer intento importante de ubicar la ciudad en el contexto regional y proyectarla hacia el futuro, según las grandes tendencias de desarrollo: Definió Escenarios y Estrategias para los hidrocarburos y energía, las telecomunicaciones, ciudad de servicios, ciudad - región, ciudad de ciencia, tecnología e innovación, centro cultural y artístico, entre otros. 


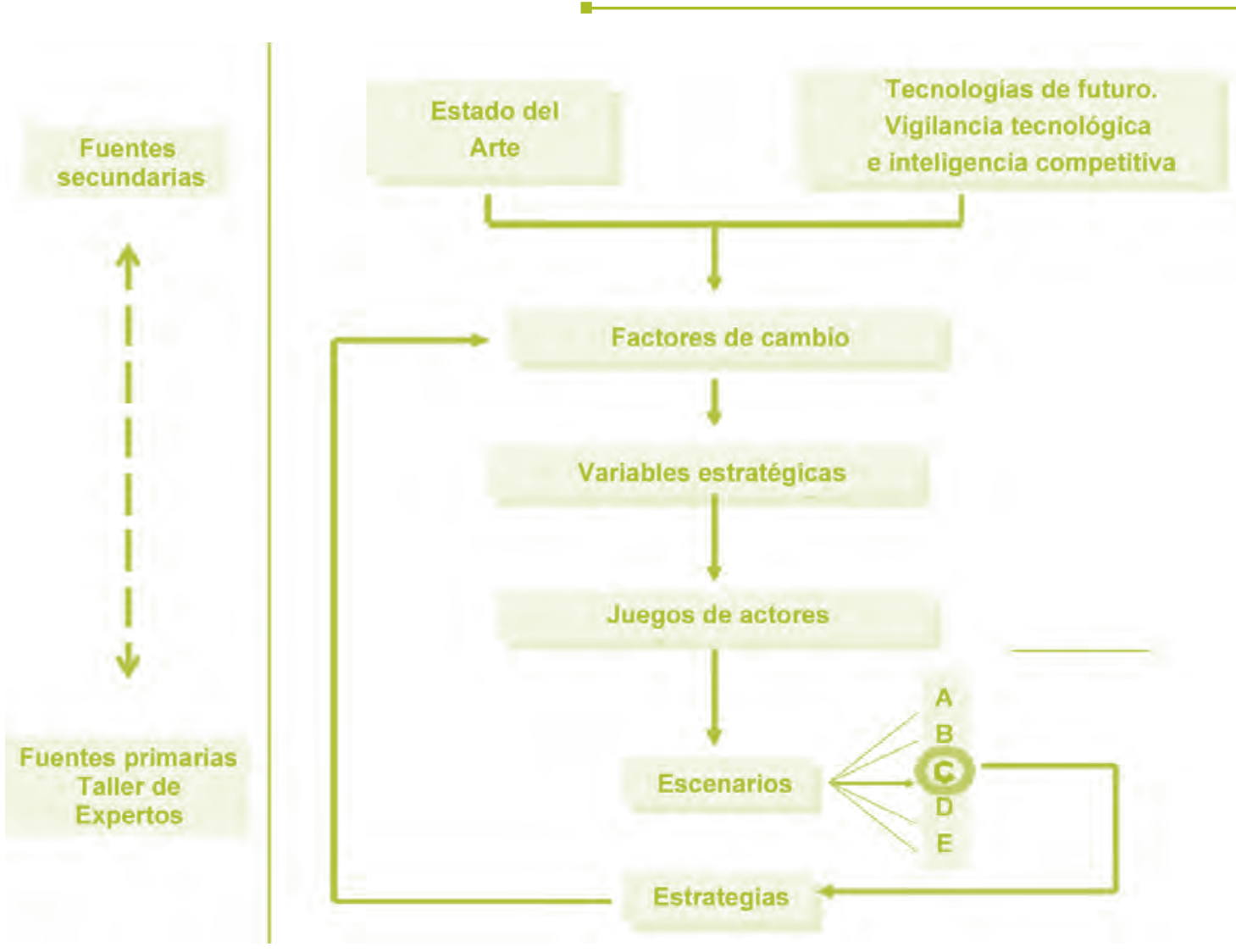

Fuente: Francisco José Mojica «Introducción a la Prospectiva Estratégica para la Competitividad Empresarial» (2010)

Gráfico 1. Modelo de prospectiva estratégica

* Los lectores interesados en conocer profundamente estas experiencias deben consultar el libro «Construyendo el Huila del 2020» recientemente editado por la Universidad Surcolombiana -Facultad de Economía y Administración- 2011.

\section{- Futuro de Paz Verde para el Huila}

Es la reflexión de mayor alcance hasta ahora prospectada en el Huila, periodo 1995-2095 (cien años). La Universidad Surcolombiana con otros Actores Sociales convocados por el Diario La Nación, sueña en la construcción de Escenarios Probables a partir de tendencias de desarrollo poblacional, los nuevos asentamientos humanos y los Ecosistemas Estratégicos, la Red Acuática y el Tiempo Libre, entre otros.

\section{- Visión HUILA 2020}

El Proyecto Huila 2020, surgió como idea de un grupo de representantes de los gremios empresariales, económicos, sociales, educativos y culturales, quienes concibieron el propósito de definir el rumbo para el Departamento del Huila, tendiente a direccionar y aprovechar las diferentes potencialidades de desarrollo regional, con el reto de visionar y construir el futuro del Huila en un horizonte de 20 años, siguiendo la experiencia del Consejo de Competitividad de Antioquia.

El principal resultado del proyecto fue la formulación de la VISIÓN HUILA 2020 en los siguientes términos: «En el 2020 el Huila será Región pacífica, emprendedora, solidaria, justa y competitiva; líder en el aprovechamiento sostenible y sustentable de la Biodiversidad, el Macizo colombiano y los demás ecosistemas estratégicos de su Territorio. Será el corazón verde y amable de Colombia, líder de una región dinámica, donde florecen los sueños de todos» (Declaración de voluntad colectiva 2001). 
En términos de esta aspiración histórica colectiva la VISIÓN 2020 identificó los siguientes escenarios: Talento Humano - Sinergia - Imaginarios - Sociedad ambiental - Energía - Mercados verdes. Así:

- Desarrollar el Talento humano (para la creatividad y la innovación). Esto se hace con educación, investigación y vigorizando la cultura.

- Desarrollar Sinergia entre los distintos Actores sociales (para el Emprendimiento y Fortalecimiento de la institucionalidad). Ganar voluntades a favor de cada escenario pero por sobretodo conseguir buena onda (ambientes de confianza y comprometer a los distintos actores sociales: A líderes y visionarios).

- Recrear y potenciar los imaginarios culturales y el mundo simbólico de la cultura huilense (creatividad terrígena y apertura a los sincretismos y mentalidades foráneas).

- El aprovechamiento sostenible y sustentable de la biodiversidad, el Macizo Colombiano y los demás ecosistemas estratégicos, sobrellevarán los principios de la Sociedad Ambiental en el sur colombiano $y$ en el resto del mundo. Promover a toda escala el reencuentro de nuevos equilibrios simbióticos con el medio natural y la cultura huilense.

- Conocer de manera juiciosa, científica y sistemática los potenciales usos y beneficios sustentables de las diversas fuentes y alternativas de energía: combustible, hidráulica, solar, cósmica, química, las fuentes globales de competitividad y desarrollo. Particularmente el beneficio de la órbita geoestacionaria para el mejoramiento de las comunicaciones y las telecomunicaciones.

- Consolidación y desarrollo de mercados verdes para productos orgánicos. Sistema agroalimentario sostenible y agroindustria (valor agregado regional) con destino a los mercados globales.

- La construcción de una Nueva Institucionalidad con sus bases de legitimidad y confianza en la sociedad civil: tejido social, proyecto ético político, gobernabilidad, conectividad.

La estrategia fundamental de este proceso articulador - regional contiene los siguientes fundamentos:
- Desarrollo territorial de la infraestructura vial, las comunicaciones - telecomunicaciones y los servicios energéticos.

- Gestión con Brasil - Venezuela y Ecuador para la culminación del corredor Amazónico - Transandino, que busca la conexión del océano Atlántico con el Pacífico, pasando por Puerto Asís - Mocoa (Putumayo) y Pitalito (de allí se abre al Huila).

- De otra parte, la terminación de la Marginal de la Selva que conecta directamente Perú - Ecuador con Venezuela, pasando por Mocoa - Florencia San Vicente del Caguán - Villavicencio.

- La vía Natagaima - Alpujarra - Colombia - La Uribe - Villavicencio, comunicará a los llanos y selvas orientales del país con la cuenca del $\mathrm{Pa}$ cífico (buscando la salida al Mar), beneficiando directamente al Huila y al Tolima.

Aclaración y sustento de la Estrategia Regional Articuladora: Debemos destacar y confirmar, de esta manera, la potente utilidad de la Metodología Prospectiva practicada en las mesas de trabajo del Proceso HUILA VISION 2020 ocurrido en el 2000, que permitió avizorar poderosas tendencias internacionales que impactarían el territorio huilense - surcolombiano como efectivamente se demostró con el recientemente conocido (en 2011) Proyecto ASPECTOS AMBIENTALES Y SOCIALES DE LAS CUENCAS ALTAS DE LOS RÍOS AMAZÓNICOS. A la fecha el Ministerio de Transporte adelanta un estudio de la cuenca del Río Amazonas, con el objetivo de elaborar el Plan de Acción Fluvial de la Cuenca Geográfica de Amazonas estableciendo las estrategias de desarrollo de sus vías fluviales. Lo anterior se espera articular con los resultados del estudio sobre el mejoramiento de la navegabilidad del Río Putumayo, enmarcado en IIRSA, el cual tiene por objeto propiciar mayor integración de la red de infraestructura de transporte de Colombia con Ecuador y Perú, así como facilitar el comercio entre los países de América del Sur.

El estudio incluirá estudios técnicos para la caracterización de la hidrovía y de sus puntos críticos, análisis ambiental y social, evaluación socio - económica, estudio de oferta y demanda de transporte de carga y pasajeros, elaboración del Plan de Acción Fluvial y marco legal. Se toma esta información de A P I - Agenda de Proyectos Prioritarios de Integración de Suramérica. Documento elaborado por IIRSA y aprobado por el Comité Coordinador 
del COSIPLAN (2011). La información relativa a los proyectos que se desarrollarán tiene como fuente la información elaborada y presentada por los países que integran la Unión de Naciones Suramericanas (UNASUR) en el marco del Consejo Suramericano de Infraestructura y Planeamiento (COSIPLAN).

Otro resultado del proyecto VISION 2020, aplicando Prospectiva, es que se logró vigorizar el desarrollo del Huila con la construcción de la nueva región, más dinámica y emprendedora, con sentido de futuro, cohesionada e integrada a su territorio, exige robustecer la institucionalidad pero también brindar posibilidades a las nuevas propuestas con sus bases de legitimidad y confianza en la sociedad civil y lo que ello aguanta.

\section{- Agenda Prospectiva de Ciencia, Tecnología e Innovación (2005-2020)}

Esta experiencia en el Huila inicia en el año 2002 con un convenio firmado entre el SENA y COLCIENCIAS y que buscaba construir colectivamente la Agenda Regional de Ciencia y Tecnología, este proceso comenzó a ejecutarse en marzo de 2003, siendo en esta época Juan de Jesús Cárdenas Chávez (2001-2003) gobernador del Departamento y los principales actores Fondo Mixto de Cultura Del Huila - FOMCULTURA, Gobernación del Huila, Cámara de Comercio de Neiva, Universidad Surcolombiana, Universidad de la Amazonía, Corpoica, Sena Regional Huila, entre otros. La experiencia finalizó en julio de 2004 con el lanzamiento oficial de la Agenda Prospectiva de $\mathrm{C}+\mathrm{T}+\mathrm{I}$ - Huila 2020 en el salón de gobernadores.

\section{El ejercicio se desarrolló en cuatro (4) Fases:}

Fase uno: Consolidación y Capacitación al Grupo Técnico y al Grupo Operativo responsables de la Formulación de la Agenda Prospectiva de Ciencia, Tecnología e Innovación con Metodología Prospectiva. Se Capacitó al Grupo Técnico en herramientas de Prospectiva.

Fase dos: Elaboración del Estado del Arte en Ciencia y Tecnología por Cada Área Estratégica. Se realizó el diagnóstico preliminar de la Ciencia y la Tecnología en el departamento del Huila.

Fase tres: Identificación y Construcción de Escenarios a partir de Talleres de Prospectiva, Consenso y Formulación de la Agenda Prospectiva de Ciencia y Tecnología. En esta fase se identificaron escenarios con el fin de definir las acciones a desarrollar en Ciencia y Tecnología en el Departamento del Huila. La Prospectiva se centró en la consulta a expertos en cada una de las áreas estratégicas definidas sobre los problemas centrales y factores de éxito que pudieran dar origen a escenarios de futuro para el movimiento de la Ciencia y la Tecnología en el Huila - 2020. De igual manera, se consultó a expertos externos, para obtener el concepto y la percepción que sobre la $\mathrm{C}+\mathrm{T}+\mathrm{l}$ existe desde otra perspectiva

Fase cuatro: Concertación de la Agenda de Compromisos por parte de los Actores Sociales (Plan de Acción y Líneas Estratégicas), con el objeto de diseñar el Plan de acción en Ciencia y Tecnología para el departamento del Huila.

Los resultados esperados al 2020 para este ejercicio son los siguientes:

- Red Huilense para el Fomento y Desarrollo de la ciencia, la tecnología y la innovación REDHCIT al 100\%.

- 36 centros locales de Innovación operando.

- 80 Huilenses con formación de Doctorado.

- 200 Huilenses con formación de Magíster.

- 25 grupos de investigación + semilleros

- Sistema regional de CTel al $100 \%$.

- Estrategia proyecto pedagógico educativo al $100 \%$.

- Proyecto pedagógico para empresarios y emprendedores al 100\%.

- 4 incubadoras de Base Tecnológica consolidadas y operando (Neiva, Pitalito, Garzón, La Plata).

- Fondo CTel reglamentado y operando (Cooperación Internacional).

- Programa Universidad + Empresa + Gobierno al 100\%.

- Red Departamental de Colegios Agropecuarios

La construcción y puesta en marcha de la RED de colegios agropecuarios del Huila, tuvo como propósito final el desarrollo integral y sostenible de las instituciones educativas involucradas, mediante procesos participativos 
y concertados de forma autónoma que posibilitara relaciones armónicas y permanentes entre ellos, en forma de Red, se realizó en 2005.

Desde esta óptica se vienen desarrollando acciones pertinentes, por parte de los actores sociales internos y externos, unificando criterios en la búsqueda de un proyecto colectivo: el trabajo en redes sociales como estrategia que contrarreste la dispersión y el quehacer individual, egoísta e improductivo de cada institución participante en la RED.

El conocerse, reconocerse, aceptarse y comprometerse en la construcción colectiva de mejores futuros sin afectar la autonomía personal e institucional, es el reto del trabajo en RED, expresión elevada y contemporánea de la «asociatividad» y la visibilidad, objetivo central del proyecto. Construir una RED sólida, como estrategia de trabajo colectivo entre los 20 colegios agropecuarios del Huila, para identificar las oportunidades, fortalezas y superar debilidades con el propósito de construir y desarrollar soluciones posibles en el largo plazo. Horizonte 2020.

La Estrategia Central de la RED de Instituciones Agropecuarias denominada «ALMA DEL HUILA» es posibilitar el afianzamiento de relaciones, procesos, acciones, intercambio de experiencias, entre los actores sociales cuya finalidad será la de satisfacer necesidades crecientes de los actores sociales en el campo educativo y productivo.

A continuación se describe el ESCENARIO APUESTA concertado:

- Red de Colegios Agropecuarios del Huila conectada a Redes Nacionales e internacionales.

- Grupos de investigación conformados en áreas de conocimiento, ciencia y tecnología + innovación.

- Actores Sociales vinculados a la Red.

- Conectividad de Nodos y Puertos.

- Empresas Autosostenibles conectadas a cadenas productivas nacionales e internacionales.

- Proyectos pedagógicos con reforma curricular articulados en Red.

\section{Plan Decenal de Cultura PDC 2011-2020}

El fortalecimiento del tejido social ha sido uno de los grandes propósitos alternativos en la actual coyuntura regional del Huila, además de hacer parte de las políticas departamentales de los últimos Planes de Desarrollo, se reconoce que ha sido el propósito clave en sucesivos Planes Decenales de Cultura (2001-2010) y (2011-2020) liderados y coordinados por las respectivas Secretarías de Cultura y Turismo del Huila y de Neiva: Reconocer en la diversidad cultural y la biodiversidad las oportunidades para el quehacer cultural, partiendo de lo local hacia lo global, promoviendo el compromiso de todos los actores sociales y gestores culturales, elevando su capacidad de gestión y fortaleciendo la identidad regional.

El Plan Decenal de Cultura del Huila (PDC HUILA 20112020) se concertó en 2010 como potente herramienta de consulta que facilita la definición de políticas, estrategias, programas y proyectos del Sistema Departamental de Cultura con visión de largo plazo, que orienta a los Actores Sociales (Institucionales y No Institucionales) y a los gestores culturales del Huila, que conduce al fortalecimiento de la actividad cultural del Huila y contribuye en la construcción general de la VISIÓN HUILA 2020.

Consecuente con el enfoque Prospectivo Territorial el proceso culminó con la Formulación del plan estratégico en el que participaron ampliamente Actores Sociales Institucionales y No Institucionales. Contiene Escenarios. Estrategias. Indicadores. Programas. Proyectos.

Como Variables estratégicas y Escenarios identificados en el Juego de Actores se pueden destacar: innovación, ciencia y tecnología, talento humano para la cultura, imaginarios, sostenibilidad, organización institucional y descentralización, patrimonio cultural tangible e intangible, los mercados verdes, entre otras.

Estrategia Transversal: «fortalecimiento de la gestión pública de la cultura y el turismo»

\section{Conclusiones generales}

- A pesar de ser la Prospectiva una ciencia nueva a nivel mundial, el Huila y la Universidad Surcolombiana han estado comprometidos con la utilización de esta nueva disciplina como eficaz instrumento para la construcción de futuro. 
- Al observar cuidadosamente las experiencias sistematizadas se puede comprobar que al Huila le ha interesado planificar colectivamente el largo plazo con la participación de la sociedad civil (Actores Sociales).

- Los sucesivos ejercicios desarrollados desde 1990 hasta el 2010 reflejan la sustitución progresiva de los paradigmas del modelo keynesiano de Desarrollo (intervencionista) por el del modelo Neoliberal (de mercados): Elevando y Fortaleciendo la capacidad de Gestión Estratégica vs. el acompañamiento del Estado.

- Los procesos permanentes y continuos de planeación se dieron continuamente pero la ejecución es muy baja comprobando que a la dirigencia regional poco 0 nada se aprecian las construcciones y aspiraciones colectivas.

- El Huila ha cumplido y ha participado activamente en todas las tareas de Planificación local, regional y nacional, en cumplimiento de requisitos y mandatos legales 0 institucionales, sin embargo los resultados de los compromisos adquiridos y sus ejecuciones permanecen públicamente desconocidas.

- Los planes prospectivos de largo plazo no han sido considerados como insumos para la formulación de los planes locales.

- La cantidad y calidad de la documentación/información que reposa en la Universidad Surcolombiana como en los distintos organismos y actores sociales que han participado directa e indirectamente en los procesos de Prospectiva Regional del Huila desde 1990, sugieren la creación de Postgrados y de un Centro de Estudios de Pensamiento Estratégico Regional en la Facultad de Economía y Administración.

- Finalmente y conforme a todas las experiencias prospectivas realizadas, se puede hacer abstracción de la «Gran Apuesta del Huila» de la siguiente manera: «En el año 2020 el Huila es el líder de la región Surcolombiana con el soporte del Sistema Regional de Ciencia, Tecnología e Innovación, de forma integral, articulada a Redes Nacionales e internacionales de conocimiento, ha fortalecido los imaginarios culturales y el mundo simbólico, es el corazón verde de Colombia, pacífico y solidario; conectado a cadenas productivas nacionales e internacionales del sector agroindustrial y minero »

\section{Referencias bibliográficas}

Amézquita, C. E. Experiencia de la Planificación Regional de la Ciencia y la Tecnología en el Huila con la Participación de la Universidad Surcolombiana: 19942004». Revista Entornos, Edición No. 19, Neiva, 2006.

Amézquita, C. E.; Santos, S. Ay Rueda, H. Construyendo el Huila 2020. Neiva: Universidad Surcolombiana, 2011.

Arapé, J. Manual de Metodologías. Tomo I: Marco teórico-conceptual. Programa de Prospectiva Tecnológica para Latinoamérica y el Caribe, 2000.

Campo, A., \& Bernal, H. El desarrollo regional en ciencia y tecnología: Situación en los países del Convenio Andrés Bello. Secretaria Ejecutiva del Convenio Andrés Bello, 1996.

Campo, A., \& Bernal, H. La cooperación multilateral en Ciencia y Tecnología y los sistemas de Ciencia y Tecnología de los países del Convenio Andrés Bello; Secretaria Ejecutiva del Convenio Andrés Bello, 1993.

Banco Nacional del Comercio Exterior S.A. Libro Blanco sobre ciencia y tecnología. Ciencia y Tecnología para una sociedad avanzada. México D.F., 1971.

Brunner, J. J. Educación: Escenarios de Futuro. Bogotá: Editorial Documentos desde Abajo, 2000.

Colciencias. Memorias del Simposio Internacional «Ciencia, Innovación y Desarrollo Regional», Bogotá, 2000.

Campo, A. Ciencia, Tecnología, Educación Superior, Gerencia Ambiental e Integración: Reflexiones. Bogotá: Editorial Solórzano S.A., 2000.

Consejo Departamental de Ciencia y Tecnología del Huila (CODECYT) -Colciencias- Universidad Surcolombiana, otros. Agenda Prospectiva de ciencia, tecnología e innovación del Huila 2020 -ARECYT- Neiva, 2004.

Corporación Huila Futuro. Estudio de Competitividad del Huila. Nuestra Riqueza Regional. (Lo que hemos construido). Neiva, 2001. 
Echarri, José M. Prospectiva y Estratégica: Una Relación Simbiótica. Recuperado de http://www.dste. ua.es/ prospectiva/Ponencias/ponencia\%20jmEcharri.doc (Último acceso julio de 2008), 2002.

Gallardo, S. La Prospectiva y su Aplicación en el proceso de la toma de decisiones en el nivel estratégico. Recuperado de http://www.egrupos.net/cgi-bin/eGruposD Mime.cgi?K9U7J9W7U7xumopxCojedevlujqejkdnoCSUYVCvthCnoqdy-qlhhyCWdgb7, 2003.

Gobernación del Huila - Universidad Surcolombiana - Comisión Regional de Ciencia y Tecnología para Centro Oriente (Colciencias). Plan de Ciencia y Tecnología del Huila. Neiva, 1998.

Godet, M. De la Anticipación a la Acción. Bogotá: Alfaomega S. A., 1999.

Godet, M. La Prospectiva Estratégica, para las empresas y los territorios. París: Dunod, 2011.

Hevia, O. R. Metodología de Escenarios: ¿Utopía o Concreción Prospectiva en las Ciencias Sociales?. Instituto de Altos Estudios de América Latina. Recuperado de http://www.iaeal.usb.ve/documentos/nro_87_90, 2000.

Jouvenel, H. Invitación a la prospectiva. Futuribles, Colección, 2004.
Medina, J. y Ortegón E. Manual de prospectiva y decisión estratégica: bases teóricas e instrumentos para América Latina y el Caribe, Instituto Latinoamericano y del Caribe de Planificación Económica y Social. Recuperado de http:/ /www.oei.es/salactsi/manual51.pdf, 2006.

Miklos, T. y Tello, M. Planeación Prospectiva: Una Estrategia para el diseño del futuro. México: Limusa, 2007.

Mojica, F. J. La Construcción del Futuro. Universidad Externado, Convenio Andrés Bello, 2006.

Mojica, F. J. Forecasting y Prospectiva dos alternativas complementarias para adelantarnos al futuro. Universidad Externado, 2008.

Ortega, F. (2006). La Prospectiva: Herramienta indispensable de planeamiento en una era de cambios. Recuperado de http://www.oei.es/salactsi/PROSPECTIVA2.PDF, 2006.

Universidad Surcolombiana. Vicerrectoría Académica. «Sistematización del Proceso de Construcción de Capacidades Regionales en Ciencia y Tecnología conseguidas a partir de la Proyección Social de la Universidad Surcolombiana en el departamento del Huila: 1994-2004». Informe Final del periodo sabático del profesor Carlos Eduardo Amézquita Parra. Neiva, 2005. 